\title{
Editorial
}

\section{The Role of $\beta$-Blockers in Cardiac Perioperative Management}

\author{
Akira Sezai, MD, PhD and Motomi Shiono, MD, PhD
}

\section{Introduction}

$\beta$-blockers which have sympathoinhibitory, cardioprotective, anti-ischemic antioxidant effects have been demonstrated as a drug with the effect of improving life prognosis in a number of large-scale studies. However, since most of the reports are studies on oral drugs, injection drugs have not been studied. There have also been no studies in patients undergoing cardiac surgery.

It has been reported that injectable $\beta$-blockers are effective for tachyarrhythmia in emergency situations because pathological conditions of sympathetic hyperactivity are often observed after invasive treatment such as surgery. Atrial fibrillation (AF) is the most common complication after cardiac surgery, with an incidence of $16 \%-85 \%$. Postoperative AF (POAF) often occurs in the early period after surgery, ${ }^{1-3)}$ and has an influence on stroke and early postoperative cardiac events, as well as on the long-term prognosis. Thus, it is important to prevent POAF.,4)

Here we will report the results of our clinical studies on the use of landiolol hydrochloride (landiolol, Ono Pharmaceutical Co., Ltd., Osaka, Japan), an injectable $\beta$-blocker, for the prevention of POAF and make a comparison with other available strategies.

\section{Cause of Atrial Fibrillation after Cardiac Surgery}

Various studies regarding the causes of POAF have been conducted, including advanced age, obesity, left atrial

$\overline{\text { Department of Cardiovascular Surgery, Nihon University School }}$ of Medicine, Tokyo, Japan

Received: December 19, 2013; Accepted: May 14, 2014 Corresponding author: Akira Sezai, MD, PhD. Department of Cardiovascular Surgery, Nihon University School of Medicine, 30-1 Oyaguchi-kamimachi, Itabashi-ku, Tokyo 173-8610, Japan Email: asezai.med@gmail.com

(C)2014 The Editorial Committee of Annals of Thoracic and Cardiovascular Surgery. All rights reserved. dimensions, cardiac dysfunction, chronic respiratory failure, renal dysfunction, fluid balance, preoperative medications, inflammation, and fibrosis, but no definitive consensus has yet been reached and various factors are involved with the occurrence ${ }^{1-5)}$ (Fig. 1). Among them, advanced age has been identified as a risk factor in a number of studies. We hypothesized that fibrosis due to aging could have an important influence on the occurrence of POAF, so we measured biomarkers of fibrosis (sialylated carbohydrate antigen KL-6 [KL-6], hyaluronic acid, and pyridinoline cross-linked telopeptide of type I collagen [I-CTP]) and atrial natriuretic peptide (ANP). This study revealed that POAF frequently occurs in cases in which the patients are at least 70 years old, the postoperative value of ANP is more than $20 \mathrm{pg} / \mathrm{dl}$, at least $30 \mathrm{U} / \mathrm{ml}$ of KL-6, at least $80 \mathrm{ng} / \mathrm{ml}$ of hyaluronic acid and at least $10 \mathrm{ng} / \mathrm{ml}$ of I-CTP, suggesting that fibrosis due to aging is largely responsible for POAF. The results of our study performed in 761 patients undergoing isolated coronary artery bypass grafting (CABG) showed that the risk factors for POAF were an age of 75 or older, chronic kidney disease, emergency surgery, cardiopulmonary bypass time $>180 \mathrm{~min}$, intraoperative carperitide non-use, intraoperative landiolol hydrochloride non-use, preoperative ARB non-use, preoperative calcium antagonist use, preoperative statin use, postoperative $\beta$-blocker non-use, and postoperative aldosterone blocker non-use, preoperative, perioperative and postoperative drug therapy also has a strong influence on the occurrence of POAF. $\left.{ }^{6}\right)$ The results of the above-mentioned study suggested that perioperative and postoperative $\beta$-blocker use was important, and emphasized the need for $\beta$-blocker therapy to suppress pathological sympathetic hyperactivity.

\section{Prevention of Postoperative Atrial Fibrillation}

Regarding the prevention of POAF, administration of amiodarone and oral administration of $\beta$-blockers are recommended in the ACC/AHA/ESC guidelines. ${ }^{5)}$ 


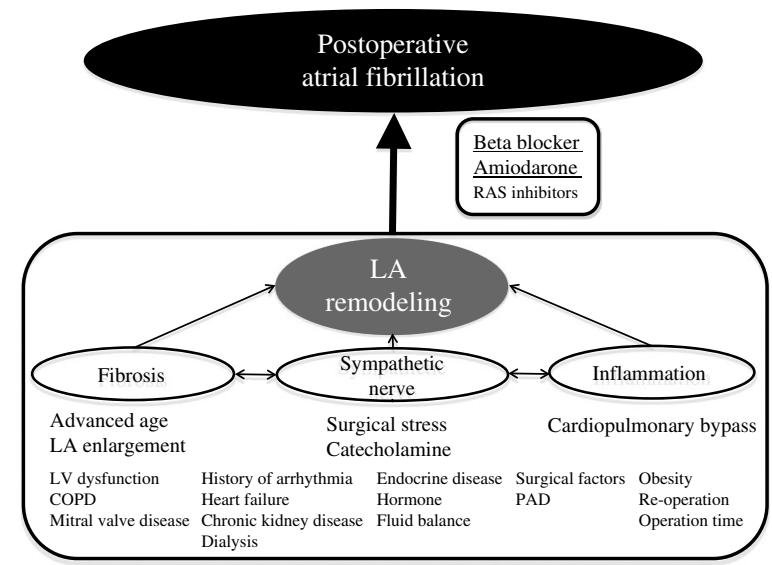

Fig. 1 Risk factors of atrial fibrillation after cardiac surgery.

However, there are no Japanese guidelines for the prevention of POAF. Crystal, et al. performed meta-analysis of 27 studies concerning $\beta$-blocker prophylaxis and reported that POAF occurred in $19 \%$ of patients receiving $\beta$-blocker groups versus $33 \%$ of untreated patients and the rate was significantly lower in the $\beta$-blocker group. ${ }^{7)}$ However, it was also reported that preoperative $\beta$-blocker administration could not prevent atrial fibrillation. ${ }^{8)}$ In a study performed at our hospital, perioperative and postoperative $\beta$-blocker therapy had a strong influence on the occurrence of POAF, but preoperative administration of $\beta$-blockers showed no preventive effect. ${ }^{6,9)}$ The results of our two studies showed that preoperative oral administration of $\beta$-blocker could not prevent POAF probably because of a small number of patients treated with $\beta$-blocker preoperatively.

Prophylaxis with oral $\beta$-blockers or amiodarone is recommended by the ACC/AHA/ESC guidelines, but the incidence of atrial fibrillation remains as high as $12.5 \%-34 \%$ when these drugs are used. ${ }^{3,78)}$ Although prophylactic administration of oral $\beta$-blockers is recommended, as mentioned above, there have been few prospective studies on injectable $\beta$-blockers and the efficacy of these drugs has not been demonstrated, probably because injectable $\beta$-blockers are more likely to cause hypotension or cardiac failure due to their negative inotropic effect. Halonen, et al. reported that intravenous administration of metoprolol was more effective than oral administration for the prevention of atrial fibrillation, with the incidence being $28.1 \%$ in the oral group versus $16.8 \%$ in the intravenous group. ${ }^{10)}$ In contrast, Maniar, et al. compared the prophylactic effect of intravenous esmolol with oral $\beta$-blockers and found no difference in the occurrence of POAF between the 2 groups, although hypotension was significantly more frequent in the intravenous esmolol group and this was a safety problem. ${ }^{11)}$ In a large-scale study comparing early intravenous administration with oral administration of metoprolol in 45852 patients after acute myocardial infarction, the incidence of non-fatal myocardial infarction and ventricular fibrillation was lower in the intravenous group than the oral group. However, the authors recommended that intravenous metoprolol should only be used when hemodynamics were stable because there was a high incidence of cardiogenic shock on the day of infection in the intravenous metoprolol group. ${ }^{12)}$ Since injectable $\beta$-blockers with a prolonged duration of action can severely compromise hemodynamics in patients who develop hypotension or bradycardia, use of these drugs during cardiac surgery is generally not recommended.

\section{New Evidence from Japan}

These injectable $\beta$-blockers are currently available in Japan, which are propranolol, esmolol, and landiolol. Propranolol has been used since the 1960s, but it has no selectivity for $\beta_{1}$ or $\beta_{2}$ receptors and prossesses intrinsic sympathomimetic activity. It has been reported that propranolol inhibits arrhythmis after non-cardiac surgery, but is associated with a high incidence of adverse reactions such as hypotension, bradycardia, and respiratory problems including asthma. ${ }^{13)}$ Because most of the conventional injectable $\beta$-blockers have no $\beta_{1}$ selectivity and a long half-life of several hours, they are not used frequently. Esmolol was developed as a superior injectable $\beta$-blocker with a short half-life and high $\beta_{1}$ selectivity. It is effective for arrhythmias associated with intraoperative hypertension, but it has been reported that there is a risk when using esmolol in patients with unstable hemodynamics who have bradycardia and hypotension. ${ }^{14)}$ Landiolol has a shorter half-life and greater $\beta_{1}$ selectivity than esmolol. It was developed in Japan and has been established as a treatment for tachyarrythmias mainly in the field of anesthesia, but landiolol has only been approved in Japan worldwide. Due to its short half-life (4 min) compared with conventional injectable $\beta$-blockers, landiolol can be used safely, while its high $\beta_{1}$ selectivity and minimal negative inotropic effect mean that there is little risk of reducing the blood pressure ${ }^{15)}$ (Table 1). We considered that landiolol was the most appropriate injectable $\beta$-blocker for patients with unstable postoperative hemodynamics, and conducted the first prospective study (PASCAL) on the use of landiolol during cardiac surgery at our hospital. ${ }^{16)}$ Patients undergoing CABG 
Table 1 Characteristics of injectable $\beta$-blockers

\begin{tabular}{lccccc}
\hline Genetic name & Product name & Half-time & $\begin{array}{c}\beta_{1} \text { selectivity } \\
\left(\beta_{1}: \beta_{2}\right)\end{array}$ & $\begin{array}{c}\text { Membrane } \\
\text { stabilizing effect }\end{array}$ & $\begin{array}{c}\text { Intrinsic } \\
\text { sympathomimetic activity }\end{array}$ \\
\hline Propranolol & Inderal & $2-6 \mathrm{~h}$ & $-(1: 1.8)$ & $(+)$ & $(-)$ \\
Metoprolol & Unapproved in Japan & $3-4 \mathrm{~h}$ & + & $(+)$ & $(-)$ \\
Esmolol & Brevibloc & $9 \mathrm{~min}$ & $+(20: 1)$ & $(+)$ & $(+)$ \\
Landiolol & Onoact & $4 \mathrm{~min}$ & $+(277: 1)$ & $(-)$ & $(-)$ \\
\hline
\end{tabular}

on cardiopulmonary bypass were randomized into two groups, i.e., a group of patients who received infusion of landiolol ( $2 \mu \mathrm{g} / \mathrm{kg} / \mathrm{min})$ for 48 hours from the time of proximal anastomosis and a group without landiolol. The incidence of POAF was significantly lower in the landiolol group than the control group (10\% vs. $34 \%$ ) and no patient discontinued the study due to hypotension, so the results demonstrated the efficacy and safety of perioperative landiolol infusion. In that study, $71 \%$ of the patients with POAF in the landiolol group developed this arrhythmia after discontinuation of landiolol. Therefore, a prospective study (BABYLON) was conducted with the duration of administration prolonged to $72 \mathrm{~h}$ and the landiolol infusion rate increased from $2 \mu \mathrm{g} / \mathrm{kg} / \mathrm{min}$ to $5 \mu \mathrm{g} /$ $\mathrm{kg} / \mathrm{min}$, as well as administration of the oral $\beta$-blocker bisoprolol from the day after surgery. ${ }^{17)}$ The biggest problem with planning this study was deciding which oral $\beta$-blocker should be used. In the ACC/AHA guidelines, lipophilic $\beta$-blockers are recommended for the treatment of chronic cardiac failure. ${ }^{18)}$ Both carvedilol and metoprolol are lipophilic drugs and have been shown to decrease cardiac events by large-scale studies in patients with acute myocardial infarction or chronic cardiac failure. However, these drugs were not selected for the study because their bradycardiac effect is weaker than that of other $\beta$-blockers based on our clinical experience. Although atenolol shown a high $\beta_{1}$ selectivity and a strong bradycardiac effects, it was not chosen either because it is water soluble and not recommended for chronic cardiac failure by the ACC/ AHA guidelines. Instead, bisoprolol was considered to be most appropriate for this study due to its high $\beta_{1}$ selectivity, potent bradycardiac effect, lipophilicity, and being recommended by the guidelines. ${ }^{18)}$ In the BABYLON study, POAF was detected in $14.7 \%$ of the landiolol monotherapy group, $9.1 \%$ of the landiolol+bisoprolol group, and $35.3 \%$ of the non- $\beta$-blocker group, with a significant difference being noted between the landiolol+ bisoprolol group and the non- $\beta$-blocker group. The abovementioned studies also demonstrated that anti-ischemic, anti-inflammatory, and sympathoinhibitory effects of $\beta$-blockers are involved in the prevention of POAF. ${ }^{16,17)}$ Based on our two trials, it was considered that sufficient effect is obtained by the method of PASCAL trial, i.e., $2 \mu \mathrm{g} / \mathrm{kg} / \mathrm{min}$ of landiolol for $48 \mathrm{~h}$. The reason is that POAF was detected in $10 \%$ of the landiolol monotherapy group in the PASCAL trial $(2 \mu \mathrm{g} / \mathrm{kg} / \mathrm{min}$ for $48 \mathrm{~h}), 14.7 \%$ of the landiolol monotherapy group in the BABYLON trial $(5 \mu \mathrm{g} / \mathrm{kg} / \mathrm{min}$ for $72 \mathrm{~h})$, and thus the incidence of POAF could not be decreased either by longer administration period or increased dosage. Instead, early switching to oral $\beta$-blockers and using $\beta$-blockers continuously were considered to be more effective.

Based on the results of these two studies, perioperative intravenous infusion of landiolol rather than preoperative oral $\beta$-blocker prophylaxis and early postoperative switching to oral $\beta$-blockers may be the most appropriate regimen for preventing POAF. In recent years, several clinical studies on the use of landiolol during cardiac surgery have been reported, ${ }^{19-23)}$ including three randomized controlled trials of landiolol for the prevention of POAF that were published following our report. ${ }^{20-22)}$ In all of these studies, POAF was shown to be significantly less frequent in the landiolol group than in the control group (Table 2).

Among the injectable $\beta$-blockers, landiolol is the only one that does not cause problems with regard to hypotension and safety, and it can be recommended for prophylaxis of atrial fibrillation after cardiac surgery.

\section{Characteristics and Future Prospects of Landiolol}

Landiolol was originally approved for the treatment of tachyarrhythmia during surgery in 2002, and was additionally approved for postoperative tachyarrythmia in 2006. In a comparative study of landiolol versus diltiazem for tachyarrythmia after cardiac surgery (JL-KNIGHT), conversion to sinus rhythm was significantly more frequent while the incidence of hypotension and bradycardia was lower in the landiolol group than the diltiazem group, 


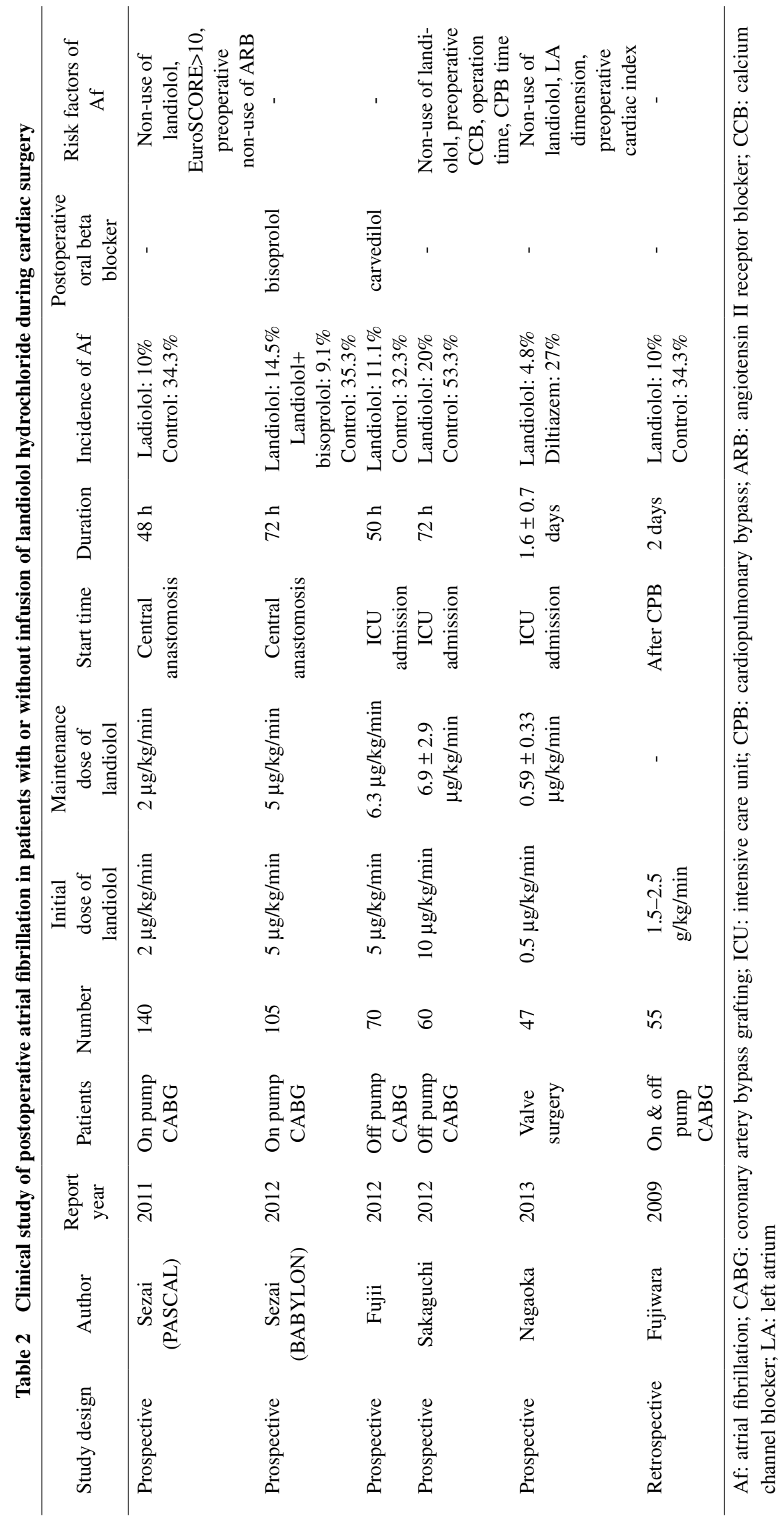


so landiolol was superior with respect to both efficacy and safety. ${ }^{24)}$ At present, landiolol is indicated for intraoperative and postoperative tachyarrythmia. Because the efficacy of oral $\beta$-blockers for cardiac failure and acute myocardial infarction has been established, a domestic multicenter study was conducted to compare landiolol and digoxin for atrial arrhythmias in patients with cardiac dysfunction. As a result of this study, the rate of achieving the primary endpoint (hear rate $<110$ /min after $2 \mathrm{~h}$ of administration and a decrease of the heart rate by $\geq 20 \%$ ) was significantly higher in the landiolol group (48\%) than in the digoxin group (13.9\%), along with fewer adverse reactions such as hypotension. ${ }^{25)}$ Since the efficacy and safety of landiolol were demonstrated by this study, it is expected that it will also be approved for treating tachyarrythmias in patients with cardiac dysfunction.

A number of large-scale studies have shown that oral $\beta$-blockers can improve the prognosis of patients with cardiac failure and acute myocardial infarction, but there is insufficient evidence reagrding injectable $\beta$-blockers. It is considered that use of injectable $\beta$-blockers will increase rapidly with the availability of landiolol, which is superior to conventional drugs in terms of safety and efficacy, and also displays a sympathoinhibitory effect, antiarrhythmic effect and anti-ischemic effect, as well as inhibition of remodeling. Oral $\beta$-blocker prophylaxis for POAF is recommended in US/EU guidelines. ${ }^{5)}$ However, there is no guideline for Japan, suggesting an urgent need to prepare a specific guideline that includes landiolol.

\section{Conclusion}

Postoperative complications have been reduced dramatically due to progress in anesthesia, myocardial protection methods, and surgical techniques. However, we consider that further improvement can be achieved by perioperative administration of drugs for organ protection, prevent of postoperative complications, and prophylaxis of atrial fibrillation. It seems that $\beta$-blocker prophylaxis may be required during cardiac surgery, while adequate multimodal therapy including RAS inhibitors and diuretics may lead to improvement of both short-term and long-term results. ${ }^{26,27)}$

\section{Disclosure Statement}

None of the authors have any conflict of interest associated with this study.

\section{References}

1) Villareal RP, Hariharan R, Liu BC, et al. Postoperative atrial fibrillation and mortality after coronary artery bypass surgery. J Am Coll Cardiol 2004; 43: 742-8.

2) Budeus $M$, Hennersdorf $M$, Perings $S$, et al. Amiodarone prophylaxis for atrial fibrillation of high-risk patients after coronary bypass grafting: a prospective, doubleblinded, placebo-controlled, randomized study. Eur Heart J 2006; 27: 1584-91.

3) Sezai A, Shiono M. Atrial fibrillation after cardiac surgery. Circ J 2013; 77: 2244-5.

4) Yaku H, Doi K. Stroke in off-pump coronary artery bypass grafting. Ann Thorac Cadiovasc Surg 2010; 16: $225-7$.

5) Fuster V, Rydén LE, Cannom DS, et al. ACC/AHA/ ESC 2006 Guidelines for the Management of Patients with Atrial Fibrillation: a report of the American College of Cardiology/American Heart Association Task Force on Practice Guidelines and the European Society of Cardiology Committee for Practice Guidelines (Writing Committee to Revise the 2001 Guidelines for the Management of Patients With Atrial Fibrillation): developed in collaboration with the European Heart Rhythm Association and the Heart Rhythm Society. Circulation 2006; 114: e257-354.

6) Sezai A, Nakata K, Mitsuru I, et al. A study on the occurrence and prevention of perioperative stroke after coronary artery bypass grafting. J Jpn Coron Assoc 2013 (in press). (in Japanese)

7) Crystal E, Connolly SJ, Sleik K, et al. Interventions on prevention of postoperative atrial fibrillation in patients undergoing heart surgery: a meta-analysis. Circulation 2002; 106: 75-80.

8) Magee MJ, Herbert MA, Dewey TM, et al. Atrial fibrillation after coronary artery bypass grafting surgery: development of a predictive risk algorithm. Ann Thorac Surg 2007; 83: 1707-12; discussion 1712.

9) Sezai A, Hata M, Niino T, et al. Study of the factors related to atrial fibrillation after coronary artery bypass grafting: a search for a marker to predict the occurrence of atrial fibrillation before surgical intervention. J Thorac Cardiovasc Surg 2009; 137: 895-900.

10) Halonen J, Hakala $T$, Auvinen $T$, et al. Intravenous administration of metoprolol is more effective than oral administration in the prevention of atrial fibrillation after cardiac surgery. Circulation 2006; 114: I1-4.

11) Maniar PB, Balcetyte-Harris N, Tamis JE, et al. Intravenous versus oral beta-blockers for prevention of post-CABG atrial fibrillation in high-risk patients identified by signal-averaged ECG: lessons of a pilot study. Card Electrophysiol Rev 2003; 7: 158-61.

12) Chen ZM, Pan HC, Chen YP, et al. Early intravenous then oral metoprolol in 45,852 patients with acute myocardial infarction: randomised placebo-controlled trial. Lancet 2005; 366: 1622-32. 
13) Bayliff CD, Massel DR, Inculet RI, et al. Propranolol for the prevention of postoperative arrhythmias in general thoracic surgery. Ann Thorac Surg 1999; 67: 182-6.

14) Sasao J, Tarver SD, Kindscher JD, et al. In rabbits, landiolol, a new ultra-short-acting beta-blocker, exerts a more potent negative chronotropic effect and less effect on blood pressure than esmolol. Can J Anaesth 2001; 48: 985-9.

15) Sugiyama A, Takahara A, Hashimoto K. Electrophysiologic, cardiohemodynamic and beta-blocking actions of a new ultra-short-acting beta-blocker, ONO-1101, assessed by the in vivo canine model in comparison with esmolol. J Cardiovasc Pharmacol 1999; 34: 70-7.

16) Sezai A, Minami K, Nakai T, et al. Landiolol hydrochloride for prevention of atrial fibrillation after coronary artery bypass grafting: new evidence from the PASCAL trial. J Thorac Cardiovasc Surg 2011; 141: 1478-87.

17) Sezai A, Nakai T, Hata M, et al. Feasibility of landiolol and bisoprolol for prevention of atrial fibrillation after coronary artery bypass grafting: a pilot study. $\mathrm{J}$ Thorac Cardiovasc Surg 2012; 144: 1241-8.

18) ACC/AHA Update Guideline for Management of Heart Failure. American College of Cardiology, American Heart Association, 2013.

19) Fujiwara H, Sakurai M, Namai A, et al. Effect of lowdose landiolol, an ultrashort-acting beta-blocker, on postoperative atrial fibrillation after CABG surgery. Gen Thorac Cardiovasc Surg 2009; 57: 132-7.

20) Fujii M, Bessho R, Ochi M, et al. Effect of postoperative landiolol administration for atrial fibrillation after off pump coronary artery bypass surgery. J Cardiovasc Surg (Torino) 2012; 53: 369-74.
21) Sakaguchi M, Sasaki Y, Hirai H, et al. Efficacy of landiolol hydrochloride for prevention of atrial fibrillation after heart valve surgery. Int Heart J 2012; 53: $359-63$.

22) Nagaoka E, Arai H, Tamura K, et al. Prevention of atrial fibrillation with ultra-low dose landiolol after off-pump coronary artery bypass grafting. Ann Thorac Cardiovasc Surg 2014; 20: 129-34.

23) Nakanishi K, Takeda S, Kim C, et al. Postoperative atrial fibrillation in patients undergoing coronary artery bypass grafting or cardiac valve surgery: intraoperative use of landiolol. J Cardiothorac Surg 2013; 8: 19 .

24) Sakamoto A, Kitakaze M, Takamoto $S$, et al. Landiolol, an ultra-short-acting $\beta_{1}$-blocker, more effectively terminates atrial fibrillation than diltiazem after open heart surgery: prospective, multicenter, randomized, open-label study (JL-KNIGHT study). Circ J 2012; 76: 1097-101.

25) Nagai R, Kinugawa K, Inoue H, et al. Urgent management of rapid heart rate in patients with atrial fibrillation/flutter and left ventricular dysfunction: comparison of the ultra-short-acting $\beta 1$-selective blocker landiolol with digoxin (J-Land Study). Circ J 2013; 77: 908-16.

26) Sezai A, Minami K, Hata M, et al. Long-term results (three-year) of emergency coronary artery bypass grafting for patients with unstable angina pectoris. Am J Cardiol 2010; 106: 511-6.

27) Sezai A, Hata M, Yoshitake I, et al. Results of emergency coronary artery bypass grafting for acute myocardial infarction: importance of intraoperative and postoperative cardiac medical therapy. Ann Thorac Cardiovasc Surg 2012; 18: 338-46. 